\title{
Einstein and Møller Energy-Momentum Complexes for a New Regular Black Hole Solution with a Nonlinear Electrodynamics Source
}

\author{
Irina Radinschi, ${ }^{1}$ Farook Rahaman, ${ }^{2}$ Theophanes Grammenos, ${ }^{3}$ and Sayeedul Islam ${ }^{2}$ \\ ${ }^{1}$ Department of Physics, Gh. Asachi Technical University, 700050 Iasi, Romania \\ ${ }^{2}$ Department of Mathematics, Jadavpur University, Kolkata, West Bengal 700 032, India \\ ${ }^{3}$ Department of Civil Engineering, University of Thessaly, 38334 Volos, Greece \\ Correspondence should be addressed to Irina Radinschi; radinschi@yahoo.com
}

Received 9 June 2016; Accepted 15 September 2016

Academic Editor: Sally Seidel

Copyright ( 2016 Irina Radinschi et al. This is an open access article distributed under the Creative Commons Attribution License, which permits unrestricted use, distribution, and reproduction in any medium, provided the original work is properly cited. The publication of this article was funded by SCOAP S $^{3}$

\begin{abstract}
A study about the energy and momentum distributions of a new charged regular black hole solution with a nonlinear electrodynamics source is presented. The energy and momentum are calculated using the Einstein and Møller energy-momentum complexes. The results show that in both pseudotensorial prescriptions the expressions for the energy of the gravitational background depend on the mass $M$ and the charge $q$ of the black hole, an additional factor $\beta$ coming from the spacetime metric considered, and the radial coordinate $r$, while in both prescriptions all the momenta vanish. Further, it is pointed out that in some limiting and particular cases the two complexes yield the same expression for the energy distribution as that obtained in the relevant literature for the Schwarzschild black hole solution.
\end{abstract}

\section{Introduction}

Energy-momentum localization plays a leading role in the theories advanced over the years in relation to General Relativity. There is a major difficulty, however, in formulating a proper definition for the energy density of gravitational backgrounds. Indeed, the key problem is the lack of a satisfactory description for the gravitational energy.

Many researchers have conducted extensive research using different methods for energy-momentum localization. Standard research methods include the use of different tools, such as super-energy tensors [1-4], quasilocal expressions [59], and the famous energy-momentum complexes of Einstein [10, 11], Landau and Lifshitz [12], Papapetrou [13], Bergmann and Thomson [14], Møller [15], Weinberg [16], and Qadir and Sharif [17]. The main problem encountered is the dependence on the reference frame of these pseudotensorial prescriptions. An alternative method used in many studies on computing the energy and momentum distributions in order to avoid the dependence on coordinates is the teleparallel theory of gravitation [18-26].

As regards pseudotensorial prescriptions, only the Møller energy-momentum complex is a coordinate independent tool. Schwarzschild Cartesian coordinates and Kerr-Schild Cartesian coordinates are useful to compute the energymomentum in the case of the other pseudotensorial definitions. Over the past few decades, despite the criticism directed against energy-momentum complexes concerning mainly the physicalness of the results obtained by them, their application has provided physically reasonable results for many spacetime geometries, more particularly for geometries in $(3+1),(2+1)$, and $(1+1)$ dimensions [27-58].

There is an agreement between the Einstein, LandauLifshitz, Papapetrou, Bergmann-Thomson, Weinberg, and Møller prescriptions, on the one hand, and the definition of the quasilocal mass advanced by Penrose [59] and developed by Tod [60] for some gravitating systems, on the other hand (see [61] for a comprehensive review). Several 
pseudotensorial definitions "provide the same results" for any metric of the Kerr-Schild class and for solutions that are more general than the Kerr-Schild class (see, e.g., the works of Aguirregabiria, Chamorro and Virbhadra, Xulu in [27-36], and Virbhadra in [62]). Furthermore, the similarity between some of the aforementioned results and those obtained by using the teleparallel theory of gravitation [63-68] cannot be overlooked. In fact, the history of energy-momentum complexes should include their definition and use, as well as the attempts for their rehabilitation [69-72].

The present work has the following structure: in Section 2 we describe the new spherically symmetric, static, charged regular black hole solution with a nonlinear electrodynamics source [73] under study. Section 3 is focused on the presentation of the Einstein and Møller energy-momentum complexes used for performing the calculations. Section 4 contains the calculations of the energy and momentum distributions. In the Discussion and Final Remarks given in Section 5, we make a brief description of the results of our investigation as well as some limiting and particular cases. Throughout the article, we use geometrized units ( $c=$ $G=1)$, the signature chosen for our purpose is $(+,-,-,-)$, and the calculations are performed using the Schwarzschild Cartesian coordinates $\{t, x, y, z\}$ for the Einstein prescription and the Schwarzschild coordinates $\{t, r, \theta, \varphi\}$ for the Møller prescription. Also, Greek indices range from 0 to 3, while Latin indices run from 1 to 3 .

\section{Description of the New Regular Black Hole Solution with a Nonlinear Electrodynamics Source}

In this section, we present a new spherically symmetric, static, charged regular black hole solution with a nonlinear electrodynamics source recently developed by Balart and Vagenas [73].

A brief but interesting discussion about the regular black hole solutions that have been obtained by coupling gravity to nonlinear electrodynamics theories is presented in [73] (see Section 1 and the references therein for more details). Further, in an interesting and similar work, the horizon entropy of a black hole is determined as a function of Komar energy and the horizon area [74].

In order to develop the new charged regular black hole solution, the authors of [73] considered the Fermi-Dirac-type distribution. For this purpose, they generalized the methodology developed in Section 2 of their paper by considering distribution functions raised to the power of a real number greater than zero. We notice that the methodology presented in Section 2 consists in constructing a general charged regular black hole metric for mass distribution functions that are inspired by continuous probability density distributions. The corresponding electric field for each black hole solution is also constructed in terms of a general mass distribution function. The metric function is given by

$$
f(r)=1-\frac{2 M}{r}\left(\frac{\sigma(\beta r)}{\sigma_{\infty}}\right)^{\beta}
$$

where $\sigma_{\infty}=\sigma(r \rightarrow \infty)$ is a normalization factor and the function $\sigma(\beta r)$ corresponds to any one of the mass functions listed in Table 1 of [73], but with the coordinate $r$ multiplied by an additional factor $\beta>0$.

The new spherically symmetric, static, charged regular black hole solution with a nonlinear electrodynamics source given by equation (29) in [73] is obtained, as we pointed out above, using the Fermi-Dirac-type distribution, and the metric function becomes now

$$
f(r)=1-\frac{2 M}{r}\left(\frac{2}{\exp \left(q^{2} / \beta M r\right)+1}\right)^{\beta} .
$$

Moreover, when $r \rightarrow \infty$ the mass function $m(r)=$ $M\left(\sigma(\beta r) / \sigma_{\infty}\right)^{\beta} \rightarrow M$. The distribution function satisfies the condition $\sigma(r) / \sigma_{\infty} \rightarrow 1$ when $r \rightarrow \infty$. This solution is a generalization of the Ayón-Beato and García black hole solution [75].

The corresponding electric field has the expression

$$
\begin{aligned}
& E(r)=\frac{q}{r^{2}} \exp \left(\frac{(1-\beta) q^{2}}{2 \beta M r}\right)\left[\operatorname{sech}\left(\frac{q^{2}}{2 \beta M r}\right)\right]^{1+\beta}[1 \\
& -\frac{q^{2}}{4 M r} \tanh \left(\frac{q^{2}}{2 \beta M r}\right) \\
& \left.+\frac{1}{4 \beta M r}\left(\frac{1-\beta}{\exp \left\{q^{2} / \beta M r\right\}+1}\right)\right] .
\end{aligned}
$$

In order to construct the extremal regular black hole metric for this example, some values of $\beta$ and the corresponding charges are listed in Table 2 of [73].

Finally, the new charged regular black hole solution with a nonlinear electrodynamics source is described by the metric

$$
d s^{2}=B(r) d t^{2}-A(r) d r^{2}-r^{2}\left(d \theta^{2}+\sin ^{2} \theta d \phi^{2}\right),
$$

with $B(r)=f(r), A(r)=1 / f(r)$.

Figure 1 shows that two horizons exist at the points where $f(r)$ meets the $(r / M)$-axis and for four different values of the parameter $\beta$. We have chosen $(q / M)^{2}=0.5$. Note that the positions of the inner and the outer horizon remain unaffected for various values of $\beta$.

\section{Einstein and Møller Energy- Momentum Complexes}

The Einstein energy-momentum complex $[10,11]$ for a $(3+$ 1)-dimensional gravitational background has the well-known expression

$$
\theta_{\nu}^{\mu}=\frac{1}{16 \pi} h_{v, \lambda}^{\mu \lambda}
$$

The superpotentials $h_{v}^{\mu \lambda}$ involved in (5) are given by

$$
h_{\nu}^{\mu \lambda}=\frac{1}{\sqrt{-g}} g_{\nu \sigma}\left[-g\left(g^{\mu \sigma} g^{\lambda \kappa}-g^{\lambda \sigma} g^{\mu \kappa}\right)\right]_{, \kappa}
$$




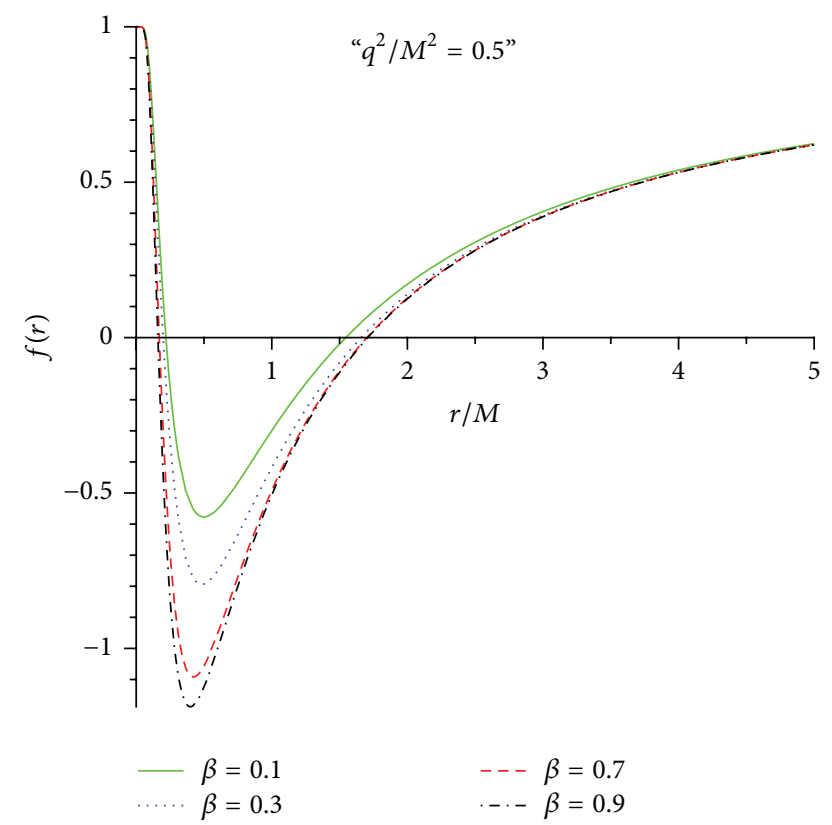

FIGURE 1: An inner and an outer horizon exist at the points where $f(r)$ meets the $(r / M)$-axis, here shown for four different values of the parameter $\beta$.

which satisfies the necessary antisymmetric property:

$$
h_{v}^{\mu \lambda}=-h_{v}^{\lambda \mu} .
$$

In the Einstein prescription, the local conservation law is respected:

$$
\theta_{\nu, \mu}^{\mu}=0
$$

Thus, the energy and momentum can be evaluated in Einstein's prescription with

$$
P_{\mu}=\iiint \theta_{\mu}^{0} d x^{1} d x^{2} d x^{3} .
$$

Here, $\theta_{0}^{0}$ and $\theta_{i}^{0}$ represent the energy and momentum density components, respectively.

Applying Gauss' theorem, the energy-momentum reads

$$
P_{\mu}=\frac{1}{16 \pi} \iint h_{\mu}^{0 i} n_{i} d S,
$$

with $n_{i}$ the outward unit normal vector over the surface $d S$. In (10), $P_{0}$ is the energy.

Concerning the expression for the Møller energymomentum complex [15], we have

$$
\mathscr{J}_{\nu}^{\mu}=\frac{1}{8 \pi} M_{v, \lambda}^{\mu \lambda}
$$

with the Møller superpotentials $M_{\nu}^{\mu \lambda}$ given by

$$
M_{\nu}^{\mu \lambda}=\sqrt{-g}\left(\frac{\partial g_{\nu \sigma}}{\partial x^{\kappa}}-\frac{\partial g_{\nu \kappa}}{\partial x^{\sigma}}\right) g^{\mu \kappa} g^{\lambda \sigma} .
$$

The Møller superpotentials $M_{v}^{\mu \lambda}$ are antisymmetric:

$$
M_{\nu}^{\mu \lambda}=-M_{\nu}^{\lambda \mu} .
$$

Like the Einstein energy-momentum complex, Møller's energy-momentum complex also satisfies the local conservation law:

$$
\frac{\partial \mathscr{J}_{v}^{\mu}}{\partial x^{\mu}}=0
$$

In (14), $\mathscr{f}_{0}^{0}$ gives the energy density and $\mathscr{F}_{i}^{0}$ represents the momentum density components.

For the Møller prescription, the energy and momentum distributions are obtained by

$$
P_{\mu}=\iiint \mathscr{J}_{\mu}^{0} d x^{1} d x^{2} d x^{3}
$$

and the energy distribution can be calculated by

$$
E=\iiint \mathscr{J}_{0}^{0} d x^{1} d x^{2} d x^{3}
$$

Again, using Gauss' theorem, one gets

$$
P_{\mu}=\frac{1}{8 \pi} \iint M_{\mu}^{0 i} n_{i} d S
$$

\section{Energy and Momentum Distribution for the New Regular Black Hole Solution with a Nonlinear Electrodynamics Source}

In order to compute the energy and momenta in the Einstein prescription, it is useful to transform the metric given by the line element (4) in Schwarzschild Cartesian coordinates applying the coordinate transformation $x=r \sin \theta \cos \varphi$, $y=r \sin \theta \sin \varphi$, and $z=r \cos \theta$. Then, the following line element is obtained:

$$
\begin{aligned}
d s^{2}= & B(r) d t^{2}-\left(d x^{2}+d y^{2}+d z^{2}\right) \\
& -\frac{A(r)-1}{r^{2}}(x d x+y d y+z d z)^{2} .
\end{aligned}
$$

The components of the superpotential $h_{\mu}^{0 i}$ in quasiCartesian coordinates for $\mu=1,2,3$ and $i=1,2,3$ are given by

$$
\begin{aligned}
& h_{1}^{01}=h_{1}^{02}=h_{1}^{03}=0, \\
& h_{2}^{01}=h_{2}^{02}=h_{2}^{03}=0, \\
& h_{3}^{01}=h_{3}^{02}=h_{3}^{03}=0 .
\end{aligned}
$$

Now, using (6), we compute the nonvanishing components of the superpotentials in the Einstein prescription and we obtain the following expressions:

$$
\begin{aligned}
& h_{0}^{01}=\frac{2 x}{r^{2}} \frac{2 M}{r}\left(\frac{2}{\exp \left(q^{2} / \beta M r\right)+1}\right)^{\beta}, \\
& h_{0}^{02}=\frac{2 y}{r^{2}} \frac{2 M}{r}\left(\frac{2}{\exp \left(q^{2} / \beta M r\right)+1}\right)^{\beta}, \\
& h_{0}^{03}=\frac{2 z}{r^{2}} \frac{2 M}{r}\left(\frac{2}{\exp \left(q^{2} / \beta M r\right)+1}\right)^{\beta} .
\end{aligned}
$$




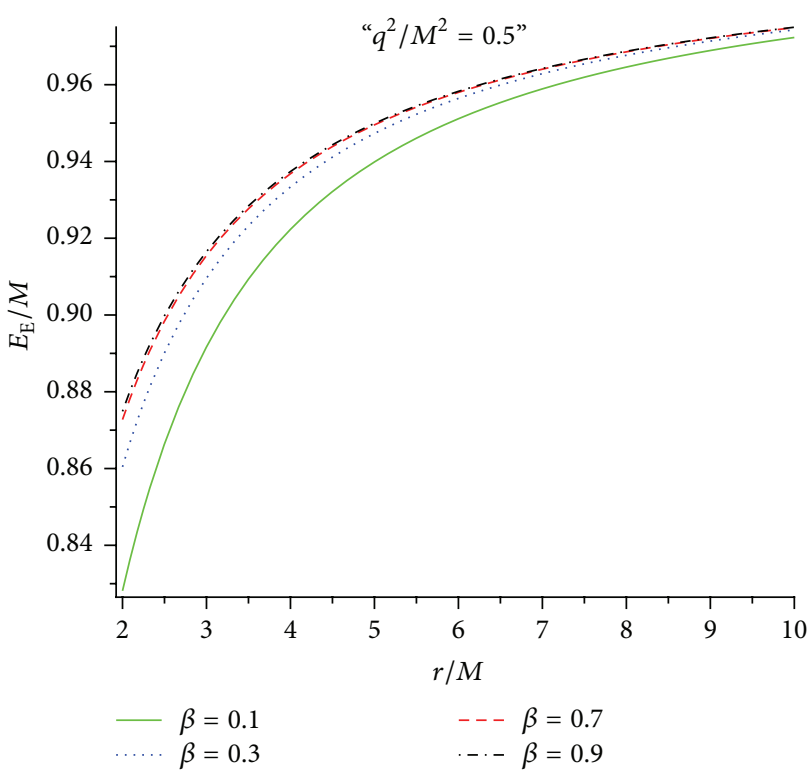

(a)

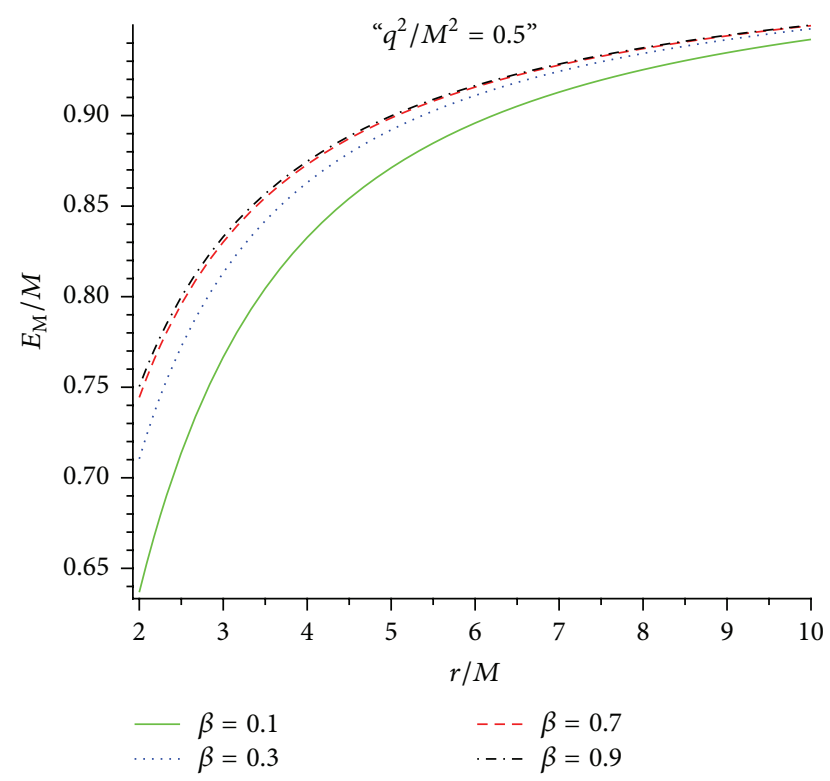

(b)

Figure 2: (a) Energy distribution computed by the Einstein prescription outside the outer horizon for four different values of $\beta$. (b) Energy distribution computed by the Møller prescription outside the outer horizon for four different values of $\beta$.

Combining the line element (18), the expression for the energy from (10), and the expressions (20) for the superpotentials, one obtains the energy distribution for the new charged regular black hole in the Einstein prescription:

$$
E_{\mathrm{E}}=M\left(\frac{2}{\exp \left(q^{2} / \beta M r\right)+1}\right)^{\beta}
$$

In order to get the momentum components, we use (10) and (19) and performing the calculations we find that all the momenta are zero:

$$
P_{x}=P_{y}=P_{z}=0 .
$$

In Figure 2(a), we plot the energy distribution in the Einstein prescription for different values of $\beta$ and $(q / M)^{2}=0.5$.

Using the Møller prescription, which is applied in Schwarzschild coordinates $\{t, r, \theta, \varphi\}$, the only nonvanishing superpotential is given by

$$
\begin{aligned}
M_{0}^{01} & =\left(\frac{2 M\left(2 /\left(\exp \left(q^{2} / \beta M r\right)+1\right)\right)^{\beta}}{r^{2}}\right. \\
& \left.-\frac{2\left(2 /\left(\exp \left(q^{2} /(\beta M r)\right)+1\right)\right)^{\beta} \times \exp \left(q^{2} / \beta M r\right) \times q^{2}}{r^{3}\left(\exp \left(q^{2} / \beta M r\right)+1\right)}\right) \\
& \cdot r^{2} \sin \theta,
\end{aligned}
$$

while all the other components of the Møller superpotential vanish.
Applying the aforementioned result for the line element (4) and using the expression (17) for the energy, we calculate the energy distribution in the Møller prescription:

$$
\begin{aligned}
E_{\mathrm{M}}= & {\left[\frac{2}{\exp \left(q^{2} / \beta M r\right)+1}\right]^{\beta} } \\
& \cdot\left[M-\frac{q^{2} \exp \left(q^{2} / \beta M r\right)}{r\left(\exp \left(q^{2} / \beta M r\right)+1\right)}\right] .
\end{aligned}
$$

Our calculations yield for all the momenta

$$
P_{r}=P_{\theta}=P_{\varphi}=0 .
$$

In Figure 2(b), we plot the energy distribution in the Møller prescription for different values of $\beta$ and $(q / M)^{2}=$ 0.5. Now, in Figure 3, we compare the expressions for energy in the Einstein and Møller prescriptions for $\beta=0.7$. Note that for large radial distances the values of energy in both prescriptions coincide.

\section{Discussion and Final Remarks}

The purpose of our paper is to study the energy-momentum for a new spherically symmetric, static and charged, regular black hole solution with a nonlinear electrodynamics source using the Einstein and Møller energy-momentum complexes. From the calculations, we conclude that in the Einstein and Møller prescriptions one obtains well-defined expressions for the energy that depend on the mass $M$ of the black hole, its charge $q$, the additional factor $\beta$, and the radial coordinate $r$. The calculations yield that for both the aforesaid used pseudotensorial prescriptions all the momenta vanish. 


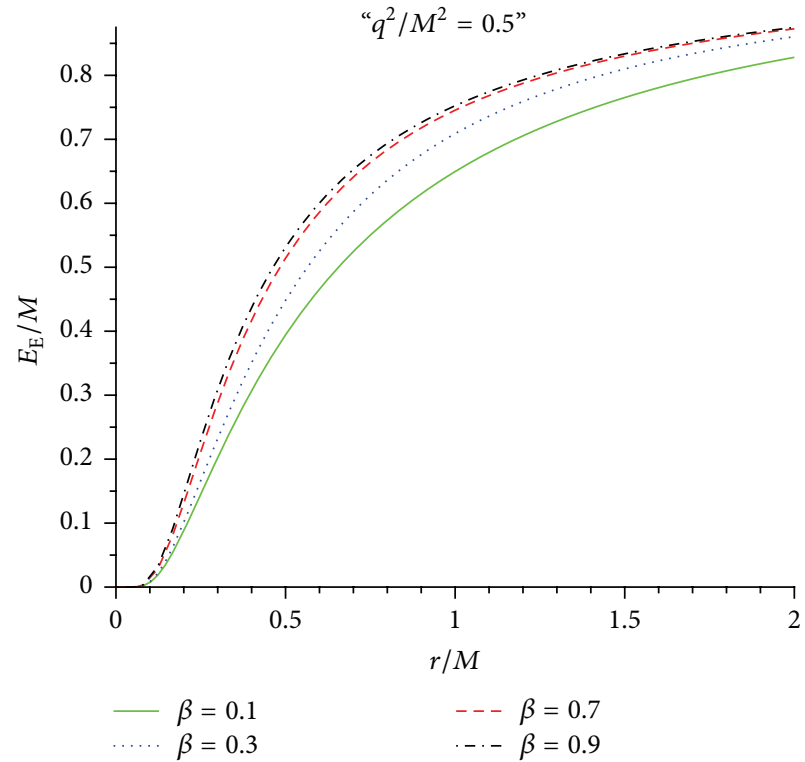

(a)

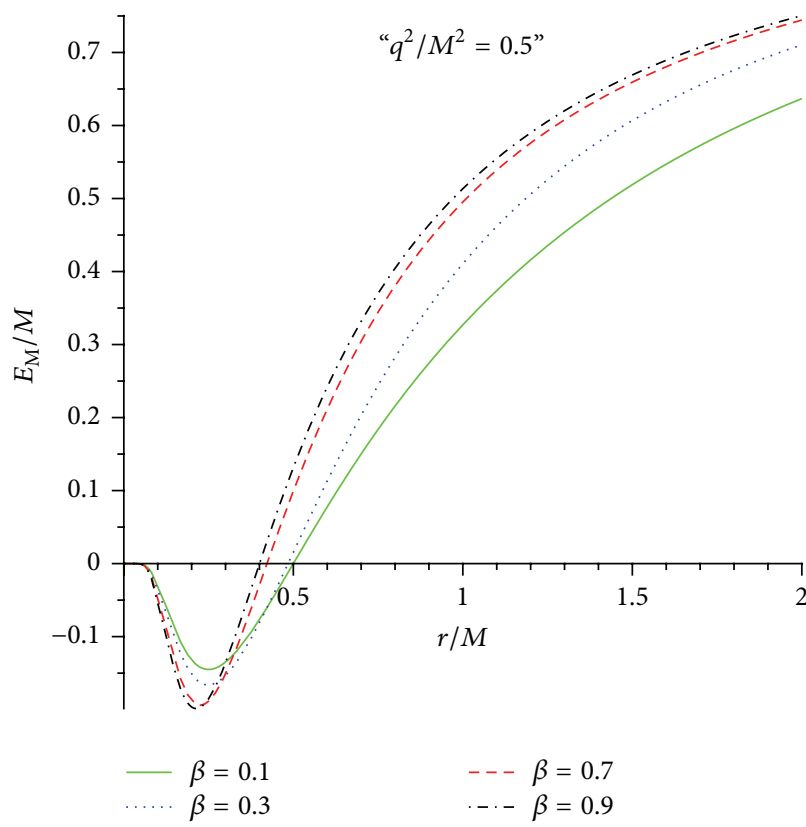

(b)

FiguRE 3: Energy distribution computed by the Einstein prescription (a) and the Møller prescription (b) near the origin for different values of $\beta$ and $(q / M)^{2}=0.5$.

TABLE 1

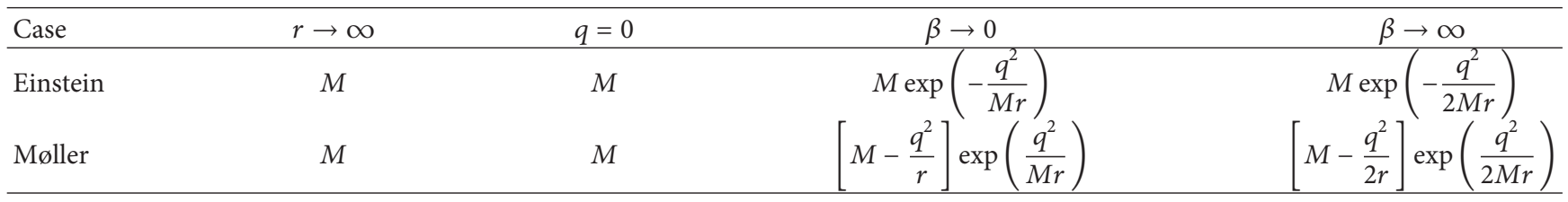

Concerning the physical meaning of the energymomentum expressions of Einstein and Møller, we study the limiting behavior of the energy for $r \rightarrow \infty, \beta \rightarrow 0$, and $\beta \rightarrow \infty$, and for the particular case $q=0$. The physically meaningful results for these limiting and particular cases are presented in Table 1.

Now, some remarks are in order. Making a comparison of the results obtained for the energy distribution with the applied Einstein and Møller definitions, we conclude that for $q=0$ and at infinity $r \rightarrow \infty$ these definitions give the same result (the ADM mass $M$ ) as that obtained for the Schwarzschild black hole solution. Moreover, this is a confirmation of Virbhadra's view point [62].

In the limiting cases $\beta \rightarrow 0$ and $\beta \rightarrow \infty$, the Einstein and Møller prescriptions provide different results. It is worth noticing that there is a difference in a factor of 2 between the exponents in the expressions for the energy. Also, we point out that although the results are different, the expressions for the energy depend on the same parameters $M, q, \beta$, and $r$. Moreover, for $\beta \rightarrow \infty$ in both prescriptions the expressions for energy are obtained for the case of equation (17) in [73]. The metric given by equation (17) in [73] represents a new well-known black hole solution in the literature that contains the metric function $f(r)=1-(2 M / r) \exp \left(-q^{2} / 2 M r\right)$. Interestingly, if in this case we consider $r \rightarrow \infty$ or $q=0$ we obtain in both prescriptions of Einstein and Møller the same expression for energy which is equal to the ADM mass $M$. In the limiting case $\beta \rightarrow 0$ with the metric function given by $f(r)=1-(2 M / r) \exp \left(-q^{2} / M r\right)$ we deduce, after some calculations, that the same results $E_{\text {Einstein }}=M$ and $E_{\text {Møller }}=M$ are also obtained by considering $r \rightarrow \infty$ or $q=0$. Table 2 summarizes these results, whereby we denote $E_{\text {Einstein }}$ by $E_{\mathrm{E}}$ and $E_{\text {Møller }}$ by $E_{\mathrm{M}}$.

A final remark regarding the behavior of energy as $r \rightarrow 0$ is deemed necessary. As one can see in Figure 3, the energy obtained by the Einstein prescription tends to zero, while the energy obtained by the Møller prescription exhibits a rather strange behavior as it takes negative values in the interval $0<$ $r<0.5$ for different values of $\beta$.

In Figure 4(a), the comparison of the two energies, here presented for a specific value of $\beta$, shows that the energies satisfy the inequality $E_{\mathrm{E}}>E_{\mathrm{M}}$ as the radial distance grows, while they tend to become equal outside the horizon for very large values of $r$ (see Figure 4(c)). 
TABLE 2

\begin{tabular}{llcc}
\hline Case & $r \rightarrow \infty$ & $q=0$ & $r \rightarrow \infty$ \\
\hline$f(r)=1-\frac{2 M}{r} \exp \left(-\frac{q^{2}}{2 M r}\right)$ & $E_{\mathrm{E}}=M$ & $E_{\mathrm{E}}=M$ & $E_{\mathrm{M}}=M$ \\
$f(r)=1-\frac{2 M}{r} \exp \left(-\frac{q^{2}}{M r}\right)$ & $E_{\mathrm{E}}=M$ & $E_{\mathrm{E}}=M$ & $E_{\mathrm{M}}=M$ \\
\hline
\end{tabular}

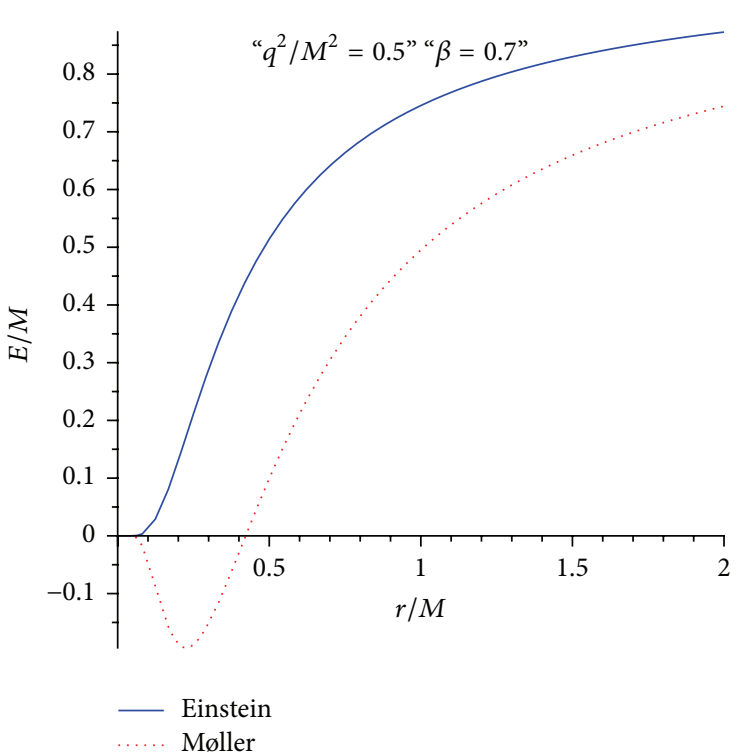

(a)

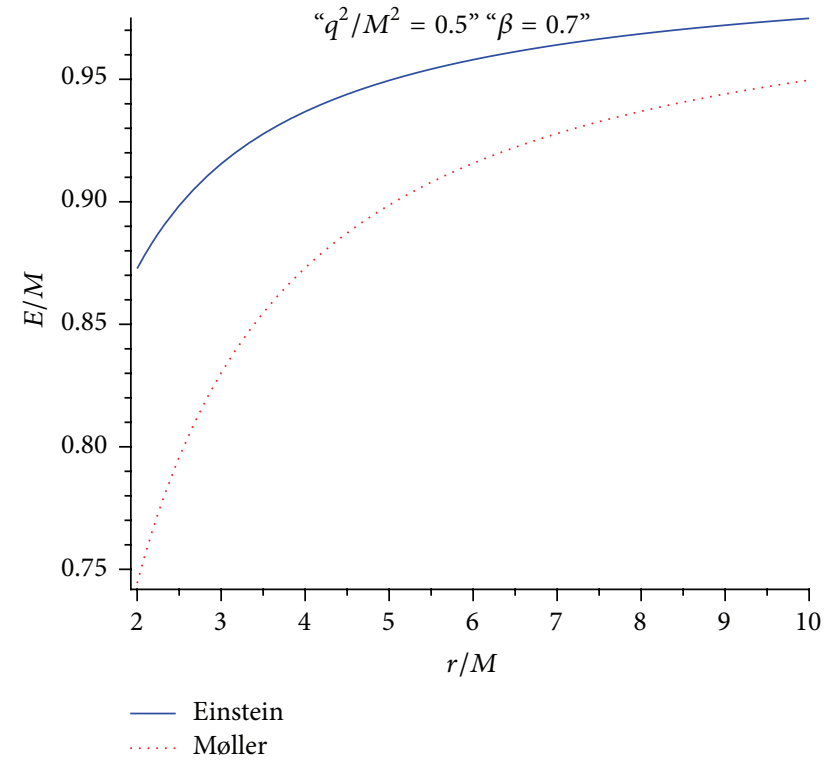

(b)

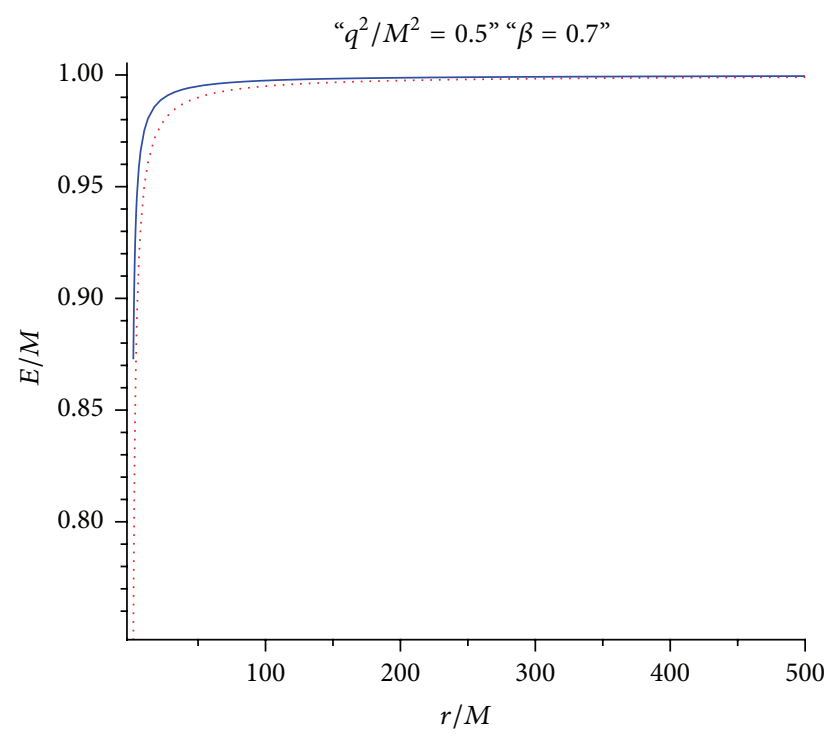

Einstein

Møller

(c)

Figure 4: For the fixed value of $\beta=0.7$ and $(q / M)^{2}=0.5$ : (a) Comparison of Einstein and Møller energies near the origin. (b) Comparison of the energy distribution performed by the Einstein and Møller prescriptions outside the outer horizon. (c) Comparison of the energy distribution performed by the Einstein and Møller prescriptions for very large values of $r$. 
The negativity of the Møller energy near the origin and, in fact, inside the inner horizon seems to be pathological and it could be attributed to the sensitivity of the Møller energy-momentum complex to the nonlinear character of the electrodynamics source. In contrast, the Einstein energy seems to be more "shielded" against this nonlinearity. We notice that a similar negativity behavior of the Møller energy has been found in [76].

In the light of the aforementioned results for the energy distribution, it is obvious that the Einstein and Møller energymomentum complexes provide well-defined and physically meaningful results and are reliable prescriptions which can be used for the study of the energy-momentum localization of gravitational backgrounds.

One can also ask what kind of astrophysical implications our results could have. It would be possible to investigate whether the effective gravitational mass is positive or negative by identifying the energy at radial distance $r$ [77] with the effective gravitational mass of the astrophysical object considered inside the region determined by the distance $r$. But it does not seem that the present case is accessible to astrophysical observations since the negative mass region is inside the inner horizon. So the present case is interesting for positive effective gravitational mass of the astrophysical object. Moreover, we would decide whether the astrophysical object could act as a convergent or as a divergent gravitational lens $[78,79]$.

Encouraged by these results, we plan, as a future perspective, to calculate the energy-momentum of this new charged regular black hole solution by using other energy-momentum complexes as well as the teleparallel equivalent. These studies can further contribute to the ongoing debate on the problem of the energy-momentum localization.

\section{Competing Interests}

The authors declare that they have no competing interests.

\section{Acknowledgments}

Farook Rahaman is grateful to the Inter-University Centre for Astronomy and Astrophysics (IUCAA), India, for providing Associateship Programme. Farook Rahaman and Sayeedul Islam are thankful to DST, Government of India, for providing financial support under SERB and INSPIRE programme.

\section{References}

[1] L. Bel, "Définition d'une densité d'énergie et d'un état de radiation totale généralisée," Comptes Rendus de l'Académie des Sciences, vol. 246, pp. 3015-3018, 1958.

[2] I. Robinson, "On the Bel-Robinson tensor," Classical and Quantum Gravity, vol. 14, no. 1, pp. A331-A333, 1997.

[3] M. A. G. Bonilla and J. M. M. Senovilla, "Some properties of the BEL and BEL-robinson tensors," General Relativity and Gravitation, vol. 29, no. 1, pp. 91-116, 1997.

[4] J. M. Senovilla, "Super-energy tensors," Classical and Quantum Gravity, vol. 17, no. 14, pp. 2799-2841, 2000.
[5] J. D. Brown and J. W. York, "Quasilocal energy and conserved charges derived from the gravitational action," Physical Review $D$, vol. 47, no. 4, p. 1407, 1993.

[6] S. A. Hayward, "Quasilocal gravitational energy," Physical Review. D. Third Series, vol. 49, no. 2, pp. 831-839, 1994.

[7] C.-M. Chen and J. M. Nester, "Quasilocal quantities for general relativity and other gravity theories," Classical and Quantum Gravity, vol. 16, no. 4, pp. 1279-1304, 1999.

[8] C.-C. M. Liu and S.-T. Yau, "Positivity of quasilocal mass," Physical Review Letters, vol. 90, no. 23, Article ID 231102, 4 pages, 2003.

[9] L. Balart, "Quasilocal energy, Komar charge and horizon for regular black holes," Physics Letters B, vol. 687, no. 4-5, pp. 280$285,2010$.

[10] A. Einstein, "On the general theory of relativity," A. Einstein, Sitzungsber. Preuss. Akad. Wiss., vol. 47, pp. 778-786, 1915, Addendum: Preuss. Akad. Wiss., vol. 47, p. 799, 1915.

[11] A. Trautman, "Conservation laws in general relativity," in Gravitation: an Introduction to Current Research, L. Witten, Ed., p. 169, John Wiley \& Sons, New York, NY, USA, 1962.

[12] L. D. Landau and E. M. Lifshitz, The Classical Theory of Fields, Pergamon Press, New York, NY, USA, 1987.

[13] A. Papapetrou, "Einstein's theory of gravitation and flat space," Proceedings of the Royal Irish Academy. Section A: Mathematical and Physical Sciences, vol. 52, pp. 11-23, 1948.

[14] P. G. Bergmann and R. Thomson, "Spin and angular momentum in general relativity," Physical Review Letters, vol. 89, pp. 400407, 1953.

[15] C. Møller, "On the localization of the energy of a physical system in the general theory of relativity," Annals of Physics, vol. 4, no. 4, pp. 347-371, 1958.

[16] S. Weinberg, Gravitation and Cosmology: Principles and Applications of General Theory of Relativity, John Wiley \& Sons, New York, NY, USA, 1972.

[17] A. Qadir and M. Sharif, "General formula for the momentum imparted to test particles in arbitrary spacetimes," Physics Letters A, vol. 167, no. 4, pp. 331-334, 1992.

[18] C. Møller, "The four-momentum of an insular system in general relativity," Nuclear Physics, vol. 57, pp. 330-338, 1964.

[19] K. Hayashi and T. Shirafuji, "New general relativity," Physical Review. D. Particles and Fields. Third Series, vol. 19, no. 12, pp. 3524-3553, 1979.

[20] J. M. Nester, L. L. So, and T. Vargas, "Energy of homogeneous cosmologies," Physical Review D, vol. 78, no. 4, Article ID 044035, 11 pages, 2008.

[21] G. G. Nashed and T. Shirafuji, "Reissner-Nordström space-time in the tetrad theory of gravitation," International Journal of Modern Physics. D. Gravitation, Astrophysics, Cosmology, vol. 16, no. 1, pp. 65-79, 2007.

[22] J. W. Maluf, F. F. Faria, and S. C. Ulhoa, "On reference frames in spacetime and gravitational energy in freely falling frames," Classical and Quantum Gravity, vol. 24, no. 10, pp. 2743-2753, 2007.

[23] J. W. Maluf, M. V. O. Veiga, and J. F. da Rocha-Neto, "Regularized expression for the gravitational energy-momentum in teleparallel gravity and the principle of equivalence," General Relativity and Gravitation, vol. 39, no. 3, pp. 227-240, 2007.

[24] A. A. Sousa, R. B. Pereira, and A. C. Silva, "Energy and angular momentum densities in a Gödel-type universe in teleparallel geometry," Gravitation and Cosmology, vol. 16, no. 1, pp. 25-33, 2010. 
[25] M. Sharif and S. Taj, "Energy contents of some non-vacuum spacetimes in teleparallel gravity," Astrophysics and Space Science, vol. 325, no. 1, pp. 75-83, 2009.

[26] Y. X. Liu, Z. H. Zhao, J. Yang, and Y. S. Duan, "The total energy-momentum of the universe in teleparallel gravity," https://arxiv.org/abs/0706.3245.

[27] K. S. Virbhadra, "Energy associated with a Kerr-Newman black hole," Physical Review D, vol. 41, no. 4, pp. 1086-1090, 1990.

[28] K. S. Virbhadra, "Energy distribution in Kerr-Newman spacetime in Einstein's as well as Møller's prescriptions," Physical Review D, vol. 42, no. 8, pp. 2919-2921, 1990.

[29] N. Rosen and K. S. Virbhadra, "Energy and momentum of cylindrical gravitational waves," General Relativity and Gravitation, vol. 25, no. 4, pp. 429-433, 1993.

[30] K. S. Virbhadra and J. C. Parikh, "A conformal scalar dyon black hole solution," Physics Letters B, vol. 331, no. 3-4, pp. 302-304, 1994.

[31] J. M. Aguirregabiria, A. Chamorro, and K. S. Virbhadra, "Energy and angular momentum of charged rotating black holes," General Relativity and Gravitation, vol. 28, no. 11, pp. 1393-1400, 1996.

[32] S. S. Xulu, "Møller energy for the Kerr-Newman metric," Modern Physics Letters A, vol. 15, no. 24, pp. 1511-1517, 2000.

[33] S. S. Xulu, "Total energy of the Bianchi type I universes," International Journal of Theoretical Physics, vol. 39, no. 4, pp. 1153-1161, 2000.

[34] S. S. Xulu, "Bergmann-Thomson energy-momentum complex for solutions more general than the Kerr-Schild class," International Journal of Theoretical Physics, vol. 46, no. 11, pp. 29152922, 2007.

[35] P. K. Sahoo, K. L. Mahanta, D. Goit et al., "Einstein energymomentum complex for a phantom black hole metric," Chinese Physics Letters, vol. 32, no. 2, Article ID 020402, 2015.

[36] S. K. Tripathy, B. Mishra, G. K. Pandey, A. K. Singh, T. Kumar, and S. S. Xulu, "Energy and momentum of bianchi type $\mathrm{VI}_{h}$ universes," Advances in High Energy Physics, vol. 2015, Article ID 705262, 8 pages, 2015.

[37] I.-C. Yang and I. Radinschi, "On the difference of energy between the Einstein and Moller prescription," Chinese Journal of Physics, vol. 42, no. 1, p. 40, 2004.

[38] I. Radinschi and T. Grammenos, “Møller's energy-momentum complex for a spacetime geometry on a noncommutative curved D3-brane," International Journal of Theoretical Physics, vol. 47, no. 5, pp. 1363-1372, 2008.

[39] I.-C. Yang, C.-L. Lin, and I. Radinschi, “The energy of regular black hole in general relativity coupled to nonlinear electrodynamics," International Journal of Theoretical Physics, vol. 48, no. 1, pp. 248-255, 2009.

[40] I. Radinschi, F. Rahaman, and A. Ghosh, "On the energy of charged black holes in generalized dilaton-axion gravity," International Journal of Theoretical Physics, vol. 49, no. 5, pp. 943-956, 2010.

[41] I. Radinschi, F. Rahaman, and A. Banerjee, "On the energy of Hořava-Lifshitz black holes," International Journal of Theoretical Physics, vol. 50, no. 9, pp. 2906-2916, 2011.

[42] I. Radinschi, F. Rahaman, and A. Banerjee, "The energy distribution of hořava-lifshitz black hole solutions," International Journal of Theoretical Physics, vol. 51, no. 5, pp. 1425-1434, 2012.

[43] M. Abdel-Megied and R. M. Gad, "Møller's energy in the Kantowski-Sachs space-time," Advances in High Energy Physics, vol. 2010, Article ID 379473, 6 pages, 2010.
[44] R. M. Gad, "On teleparallel version of stationary axisymmetric solutions and their energy contents," Astrophysics and Space Science, vol. 346, no. 2, pp. 553-557, 2013.

[45] T. Bringley, "Energy and momentum of a stationary beam of light," Modern Physics Letters A, vol. 17, no. 3, pp. 157-161, 2002.

[46] I. Radinschi, F. Rahaman, and U. F. Mondal, "Energy distribution for non-commutative radiating Schwarzschild black holes," International Journal of Theoretical Physics, vol. 52, no. 1, pp. 96104, 2013.

[47] M. Sukenik and J. Sima, "Energy and momentum in expansive nondecelerative universe," http://arxiv.org/abs/gr-qc/0101026.

[48] M. Sharif and T. Fatima, "Energy distribution associated with static axisymmetric solutions," Astrophysics and Space Science, vol. 302, no. 1-4, pp. 217-224, 2006.

[49] M. Sharif and M. Azam, "Energy-momentum distribution: some examples," International Journal of Modern Physics A, vol. 22, no. 10, pp. 1935-1951, 2007.

[50] P. Halpern, "Energy of the taub cosmological solution," Astrophysics and Space Science, vol. 306, no. 4, pp. 279-283, 2006.

[51] E. C. Vagenas, "Energy distribution in 2d stringy black hole backgrounds," International Journal of Modern Physics A, vol. 18, no. 31, pp. 5781-5794, 2003.

[52] E. C. Vagenas, "Effective mass of a radiating charged particle in Einstein's universe," Modern Physics Letters A, vol. 19, no. 3, pp. 213-222, 2004.

[53] E. C. Vagenas, "Energy distribution in a btz black hole spacetime," International Journal of Modern Physics D, vol. 14, no. 03n04, pp. 573-585, 2005.

[54] E. C. Vagenas, "Energy distribution in the dyadosphere of a reissner-nordström black hole in møller's prescription," Modern Physics Letters A, vol. 21, no. 25, pp. 1947-1956, 2006.

[55] T. Multamaki, A. Putaja, E. C. Vagenas, and I. Vilja, "Energymomentum complexes in $f(R)$ theories of gravity," Classical and Quantum Gravity, vol. 25, no. 7, Article ID 075017, 2008.

[56] L. Balart, "Energy distribution of (2+1)-dimensional black holes with nonlinear electrodynamics," Modern Physics Letters A, vol. 24, no. 34, pp. 2777-2785, 2009.

[57] A. M. Abbassi, S. Mirshekari, and A. H. Abbassi, "Energymomentum distribution in static and nonstatic cosmic string space-times," Physical Review D, vol. 78, no. 6, Article ID 064053, 2008.

[58] J. Matyjasek, "Some remarks on the Einstein and Møller pseudotensors for static and spherically-symmetric configurations," Modern Physics Letters A: Particles and Fields, Gravitation, Cosmology, Nuclear Physics, vol. 23, no. 8, pp. 591-601, 2008.

[59] R. Penrose, "Quasi-local mass and angular momentum in general relativity," Proceedings of the Royal Society of London A: Mathematical, Physical and Engineering Sciences, vol. 381, no. 1780 , pp. 53-63, 1982.

[60] K. P. Tod, "Some examples of Penrose's quasilocal mass construction," Proceedings of the Royal Society. London. Series A. Mathematical, Physical and Engineering Sciences, vol. 388, no. 1795, pp. 457-477, 1983.

[61] L. B. Szabados, "Quasi-local energy-momentum and angular momentum in general relativity," Living Reviews in Relativity, vol. 12, article 4, 2009.

[62] K. S. Virbhadra, "Naked singularities and Seifert's conjecture," Physical Review. D. Third Series, vol. 60, no. 10, Article ID 104041, 1999.

[63] G. G. L. Nashed, "Kerr-Newman solution and energy in teleparallel equivalent of Einstein theory," Modern Physics Letters A, vol. 22, no. 14, pp. 1047-1056, 2007. 
[64] G. G. L. Nashed, “Møller's energy of Kerr-NUT metric," Chinese Physics Letters, vol. 25, no. 4, pp. 1202-1204, 2008.

[65] G. G. Nashed, "Energy and angular momentum of general fourdimensional stationary axi-symmetric space-time in teleparallel geometry," International Journal of Modern Physics A: Particles and Fields. Gravitation. Cosmology. Nuclear Physics, vol. 23, no. 12, pp. 1903-1918, 2008.

[66] G. G. L. Nashed, "Brane world black holes in teleparallel theory equivalent to general relativity and their Killing vectors, energy, momentum and angular momentum," Chinese Physics B, vol. 19, no. 2, Article ID 020401, 2010.

[67] G. G. Nashed, "Energy of spherically symmetric space-times on regularizing teleparallelism," International Journal of Modern Physics A. Particles and Fields. Gravitation. Cosmology, vol. 25, no. 14, pp. 2883-2895, 2010.

[68] M. Sharif and A. Jawad, "Energy contents of some well-known solutions in teleparallel gravity," Astrophysics and Space Science, vol. 331, no. 1, pp. 257-263, 2011.

[69] C.-C. Chang, J. M. Nester, and C.-M. Chen, "Pseudotensors and quasilocal energy-momentum," Physical Review Letters, vol. 83, no. 10, pp. 1897-1901, 1999.

[70] C. M. Chen and J. M. Nester, "A Symplectic Hamiltonian derivation of quasilocal energy momentum for GR," Gravitation and Cosmology, vol. 6, pp. 257-270, 2000.

[71] L. L. So, J. M. Nester, and H. Chen, "Classical pseudotenors and positivity in small regions," in Gravitation and Astrophysics. Proceedings, 7th Asia-Pacific International Conference, J. M. Nester, C.-M. Chen, and J.-P. Hsu, Eds., p. 356, World Scientific, 2007.

[72] J. M. Nester, C. M. Chen, J.-L. Liu, and G. Sun, "A reference for the covariant hamiltonian boundary term," in Relativity and Gravitation-100 years after Einstein in Prague, J. Bičák and T. Ledvinka, Eds., pp. 177-184, Springer, Berlin, Germany, 2014.

[73] L. Balart and E. C. Vagenas, "Regular black holes with a nonlinear electrodynamics source," Physical Review D, vol. 90, no. 12, Article ID 124045, 2014.

[74] H. Culetu, "On a regular charged black hole with a nonlinear electric source," International Journal of Theoretical Physics, vol. 54, no. 8, pp. 2855-2863, 2015.

[75] E. Ayón-Beato and A. García, "New regular black hole solution from nonlinear electrodynamics," Physics Letters B, vol. 464, no. 1-2, pp. 25-29, 1999.

[76] I. Radinschi, F. Rahaman, Th. Grammenos, A. Spanou, and S. Islam, "Energy distribution of a regular black hole solution in einstein-nonlinear electrodynamics," Advances in Mathematical Physics, vol. 2015, Article ID 530281, 12 pages, 2015.

[77] J. M. Cohen and F. de Felice, "The total effective mass of the Kerr-Newman metric," Journal of Mathematical Physics, vol. 25, no. 4, pp. 992-994, 1984.

[78] K. S. Virbhadra and C. R. Keeton, "Time delay and magnification centroid due to gravitational lensing by black holes and naked singularities," Physical Review D, vol. 77, Article ID 124014, 2008.

[79] K. S. Virbhadra, "Relativistic images of Schwarzschild black hole lensing," Physical Review D, vol. 79, no. 8, Article ID 083004, 2009. 

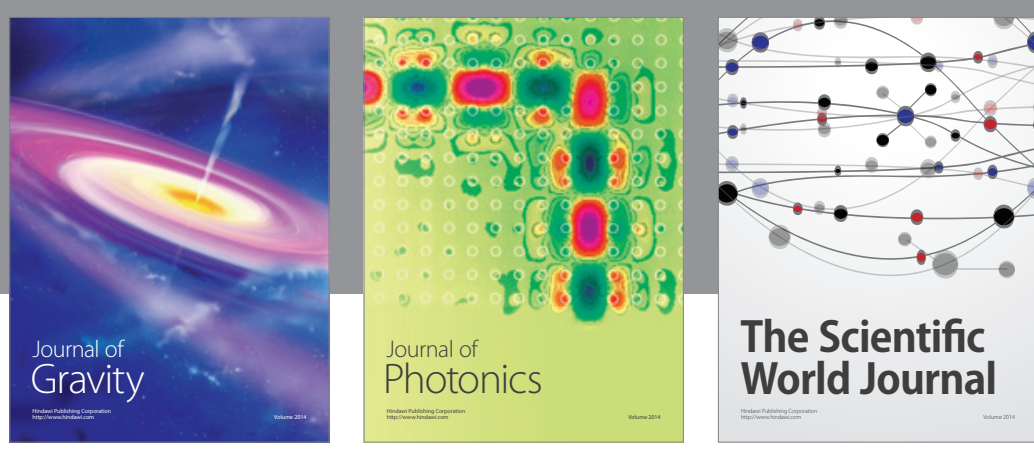

The Scientific World Journal
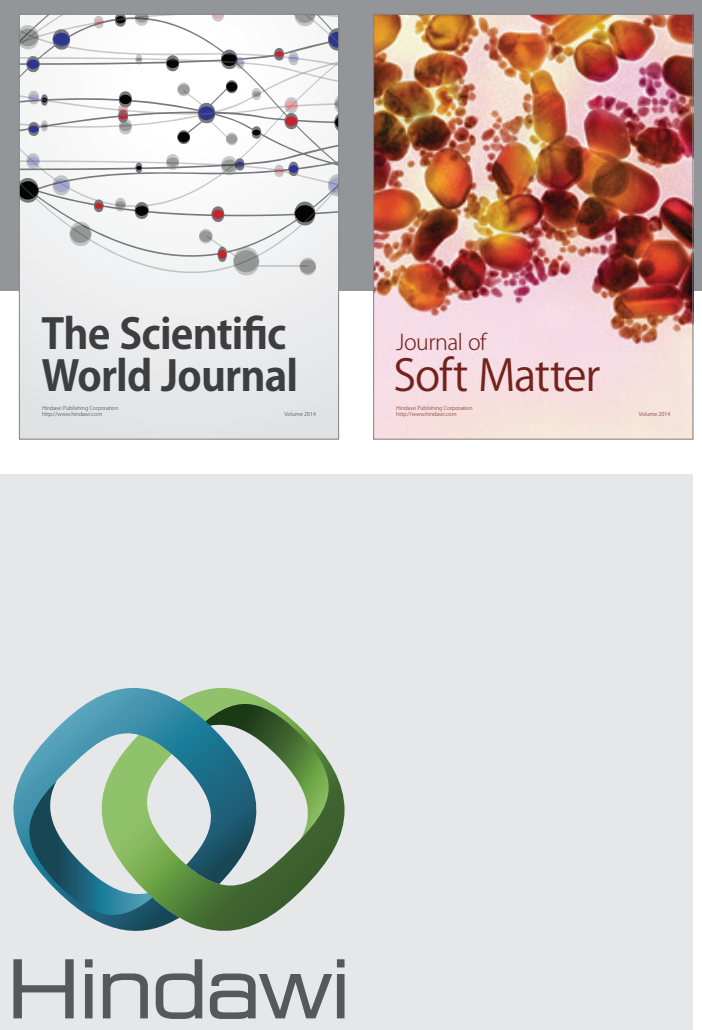

Submit your manuscripts at

http://www.hindawi.com

nternational Journal of

Statistical Mechanics
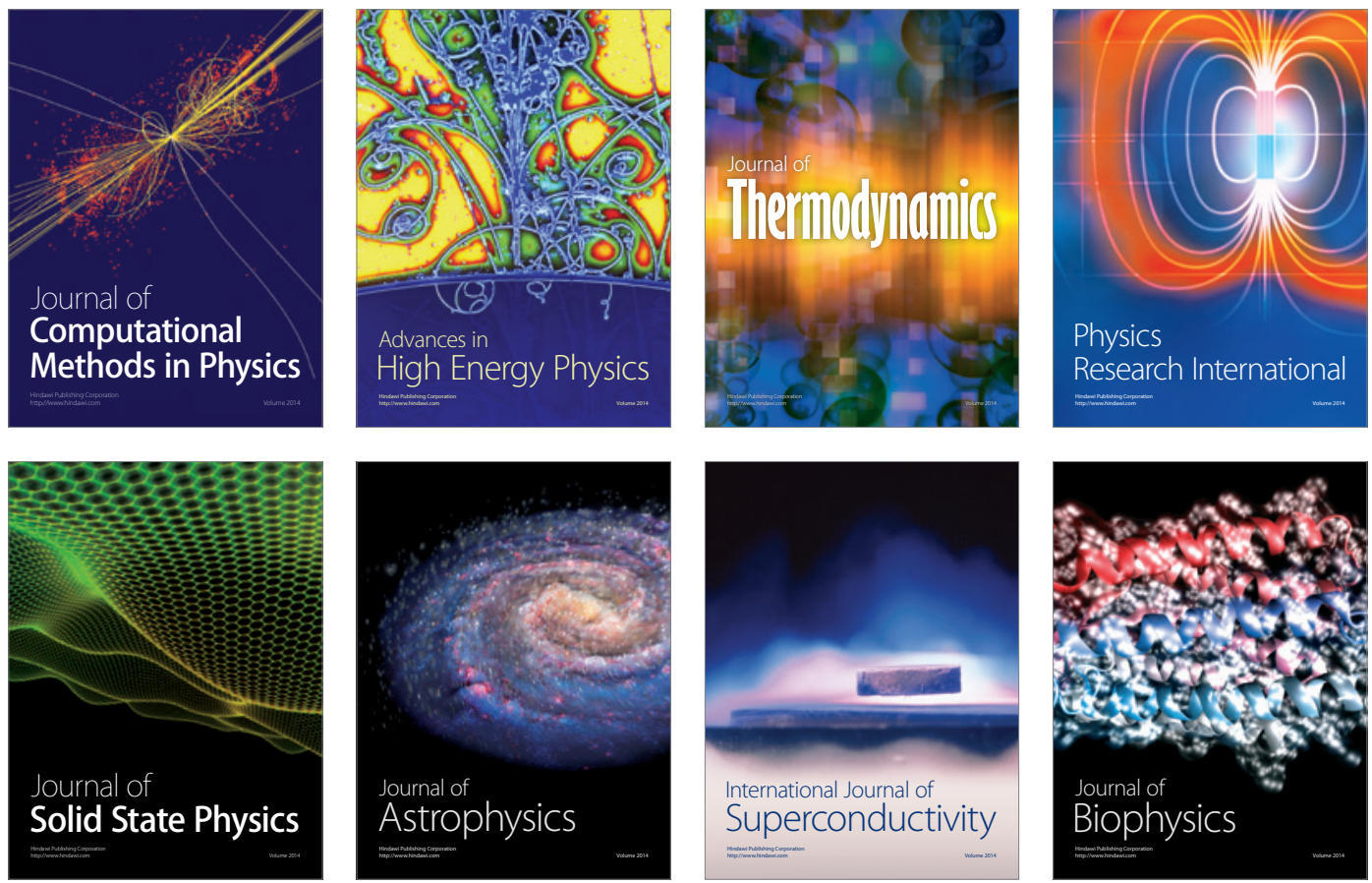
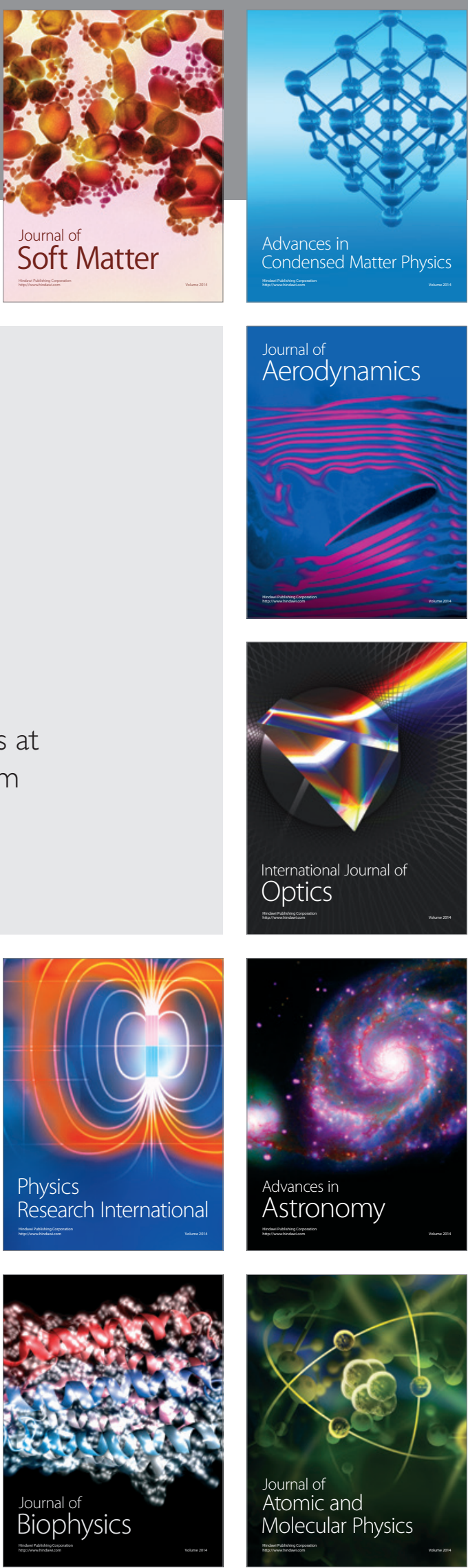